\title{
Ultrathin $\mathrm{Fe}_{3} \mathrm{O}_{4}$ epitaxial films on wide bandgap $\mathrm{GaN}(0001)$
}

\author{
P. K. J. Wong, ${ }^{1, *}$ W. Zhang, ${ }^{1}$ X. G. Cui, ${ }^{2}$ Y. B. Xu, ${ }^{1, \dagger}$ J. Wu, ${ }^{3}$ Z. K. Tao, ${ }^{2}$ X. Li, ${ }^{2}$ Z. L. Xie, ${ }^{2}$ R. Zhang, ${ }^{2}$ and G. van der Laan ${ }^{4}$ \\ ${ }^{1}$ Spintronics and Nanodevice Laboratory, Department of Electronics, University of York, York YO10 5DD, United Kingdom \\ ${ }^{2}$ Jiangsu Provincial Key Laboratory of Advanced Photonic and Electronic Materials and Department of Physics, Nanjing University, \\ Nanjing 210093, People's Republic of China \\ ${ }^{3}$ Department of Physics, University of York, York, YO10 5DD, United Kingdom \\ ${ }^{4}$ Diamond Light Source, Chilton, Didcot OX11 ODE, United Kingdom
}

(Received 3 September 2009; revised manuscript received 19 November 2009; published 20 January 2010)

\begin{abstract}
Ultrathin films of magnetite $\left(\mathrm{Fe}_{3} \mathrm{O}_{4}\right)$ have been grown epitaxially on wurtzite wide bandgap semiconductor $\mathrm{GaN}(0001)$ surfaces using molecular-beam epitaxy. Reflection high-energy electron-diffraction patterns show a (111) orientation of the $\mathrm{Fe}_{3} \mathrm{O}_{4}$ films and in-plane epitaxial relationship of $\langle 1 \overline{1} 0\rangle_{\mathrm{Fe}_{3} \mathrm{O}_{4}} \|\langle 11 \overline{2} 0\rangle_{\mathrm{GaN}}$ and $\langle 11 \overline{2}\rangle_{\mathrm{Fe}_{3} \mathrm{O}_{4}} \|\langle 1 \overline{1} 00\rangle_{\mathrm{GaN}}$ with the $\mathrm{GaN}(0001)$. X-ray photoelectron spectroscopy and $\mathrm{x}$-ray magnetic circular dichroism confirm the growth of stoichiometric $\mathrm{Fe}_{3} \mathrm{O}_{4}$, instead of $\gamma-\mathrm{Fe}_{2} \mathrm{O}_{3}$. The magnetic hysteresis loops and saturation magnetization $M_{s}$ obtained by superconducting quantum interference device at room temperature show fast saturation of the $\mathrm{Fe}_{3} \mathrm{O}_{4}$ films with the magnetization close to that of the bulk single-crystal value. In-plane magnetoresistance (MR) measurements reveal negligibly small MR effects, further indicating that the films are free from antiphase boundaries.
\end{abstract}

DOI: 10.1103/PhysRevB.81.035419

PACS number(s): 75.70.-i, 85.75.-d

\section{INTRODUCTION}

One of the major challenges in developing next generation spintronic devices, which integrate spin with semiconductors, is the synthesis of high quality magnetic materials with Curie temperatures $\left(T_{C}\right)$ above room temperature (RT), large spin polarization at the Fermi level $\left(E_{F}\right)$, and matched impedance between magnetic and semiconductor materials. ${ }^{1,2}$ Among the wide range of magnetic materials including ferromagnetic transition metals, dilute magnetic semiconductors, ${ }^{3,4}$ Heusler alloys, ${ }^{5,6}$ and half-metallic magnetic oxides, ${ }^{7}$ a highly relevant candidate perfectly matching the above criteria is $\mathrm{Fe}_{3} \mathrm{O}_{4}$. Half-metallic $\mathrm{Fe}_{3} \mathrm{O}_{4}$ is of great current interest as a RT spin injector due to its predicted half-metallicity, ${ }^{8}$ strong measured spin polarization approaching $100 \%$ at $E_{F},{ }^{9}$ and high $T_{C}$ of $858 \mathrm{~K}$, well in excess of RT. To date, epitaxial growth of $\mathrm{Fe}_{3} \mathrm{O}_{4}$ films on a variety of conventional semiconductors, such as GaAs, ${ }^{10,11}$ InAs, ${ }^{12}$ and $\mathrm{Si}^{13}$ has been achieved, whereas possible growth on wide bandgap semiconductors, such as $\mathrm{GaN}$, a technologically important material for fabrication of optoelectronic devices, ${ }^{14,15}$ and high power transistors, ${ }^{16}$ remains essentially unexplored. Recent GaN-based spin relaxation measurements yield spin lifetimes of $\sim 20 \mathrm{~ns}$ at $5 \mathrm{~K} .{ }^{17}$ Theoretical calculations predicted that the spin lifetime in pure $\mathrm{GaN}$ is about three orders of magnitude larger than in GaAs for all temperatures. ${ }^{18}$ These encouraging reports indicate that the inherent ability of GaN to sustain a long spin lifetime is greatly beneficial to device fabrication where spin transport at large length scales is required. It is, therefore, anticipated that the combination of half-metallic $\mathrm{Fe}_{3} \mathrm{O}_{4}$ and wide bandgap GaN could be one of the most promising magneticsemiconductor hybrid systems for development of future spintronic devices.

$\mathrm{Fe}_{3} \mathrm{O}_{4}$ is a mixed-valence $3 d$ transition-metal oxide that has an inverse spinel structure with a lattice constant of $8.397 \AA$. The tetrahedral sites of the spinel structure are en- tirely occupied by $\mathrm{Fe}^{3+}$, whereas the octahedral sites are occupied by both $\mathrm{Fe}^{2+}$ and $\mathrm{Fe}^{3+}$ cations. For heteroepitaxy of $\mathrm{Fe}_{3} \mathrm{O}_{4}$ on wurtzite $\mathrm{GaN}(0001)$, the $\{111\}$ faces of $\mathrm{Fe}_{3} \mathrm{O}_{4}$ offers the best atomic registry. Figure 1 depicts schematically two possible atomic arrangements with each rotated $30^{\circ}$ to the other, assuming that the $\mathrm{Fe}_{3} \mathrm{O}_{4}(111)$ film is stabilized on the (0001) plane of GaN in a hexagon-on-hexagon manner. In terms of lattice matching, configuration (1) in Fig. 1(b), i.e., $\mathrm{Fe}_{3} \mathrm{O}_{4}(111)\langle 1 \overline{1} 0\rangle \| \mathrm{GaN}(0001)\langle 11 \overline{2} 0\rangle$, gives a mismatch of $-6.5 \%$ along $\langle 11 \overline{2} 0\rangle$ and $\langle 1 \overline{1} 00\rangle$ directions of $\mathrm{GaN}(0001)$, whereas $-19.1 \%$ and $7.9 \%$ for configuration (2), with $\mathrm{Fe}_{3} \mathrm{O}_{4}(111)\langle 11 \overline{2}\rangle \| \mathrm{GaN}(0001)\langle 11 \overline{2} 0\rangle$, given the lattice constants of $\mathrm{Fe}_{3} \mathrm{O}_{4}\left(a_{\mathrm{Fe}_{3} \mathrm{O}_{4}}\right)$ and $\mathrm{GaN}\left(a_{\mathrm{GaN}}\right)$, are 8.396 and $3.189 \AA,^{19}$ respectively. In accordance, it is predicted that the former provides a crystallographically more favorable arrangement of the heterostructure.

In this work, we have investigated the epitaxial growth of $\mathrm{Fe}_{3} \mathrm{O}_{4}$ ultrathin films on $\mathrm{GaN}(0001)$ by molecular-beam epitaxy (MBE). The structural and magnetic properties of this

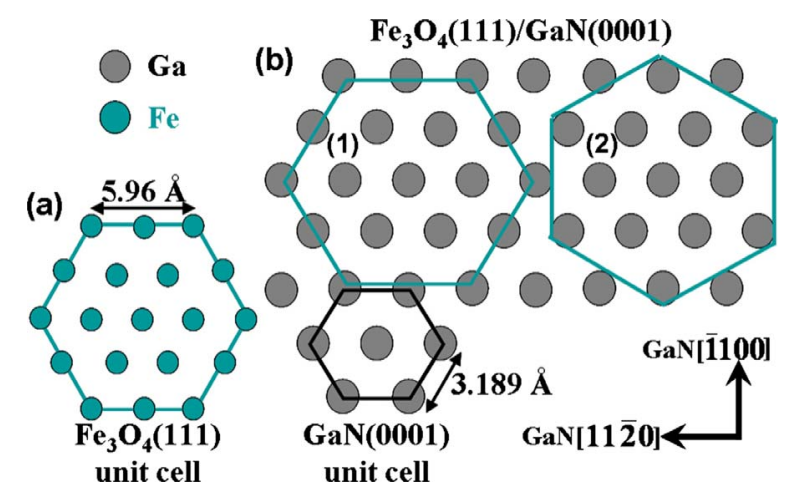

FIG. 1. (Color online) The unit cell of $\mathrm{Fe}_{3} \mathrm{O}_{4}(111)$ and $\mathrm{GaN}(0001)$ is shown, respectively, in (a) and (b). Two possible atomic arrangements of $\mathrm{Fe}_{3} \mathrm{O}_{4}(111)$ on $\mathrm{GaN}(0001)$ are also schematically depicted in (b). 


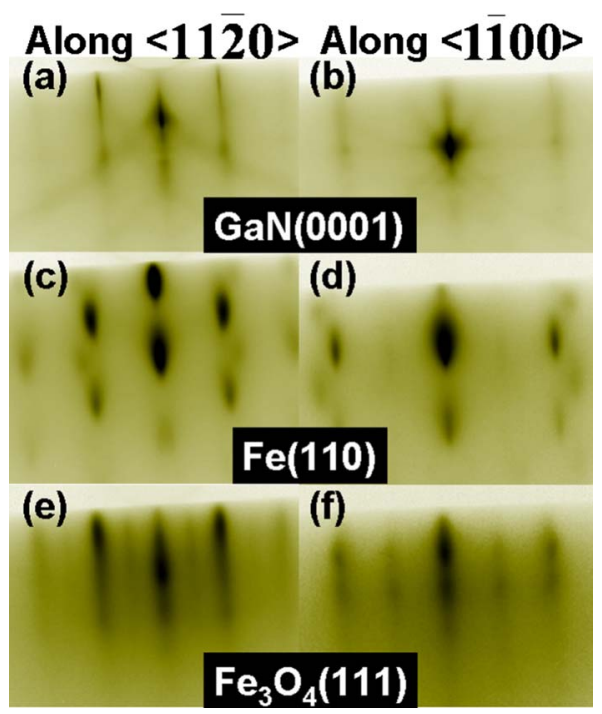

FIG. 2. (Color online) RHEED patterns taken from the $\mathrm{GaN}(0001)$ substrate, $\mathrm{Fe}(110)$, and $\mathrm{Fe}_{3} \mathrm{O}_{4}(111)$ layers with a beam energy of $10 \mathrm{kV}$ : (a) and (b) GaN surface after in situ annealing; (c)-(d) after growth of $\mathrm{Fe}(110)$ at RT; (e)-(f) after oxidation of $\mathrm{Fe}(110)$.

new hybrid material have been thoroughly studied by reflection high-energy electron diffraction (RHEED), x-ray photoelectron spectroscopy (XPS), x-ray magnetic circular dichroism (XMCD), superconducting quantum interference device (SQUID), and magnetoresistance (MR) measurements. To our knowledge, there have been no previous reports of single-crystal $\mathrm{Fe}_{3} \mathrm{O}_{4}$ growth on $\mathrm{GaN}$ and the present work can have an impact on developing and understanding this class of hybrid spintronic structure.

\section{EXPERIMENTAL DETAILS}

The $2 \mu \mathrm{m}$ thick GaN films used in this study have been prepared by metal-organic chemical vapor deposition with a $20 \mathrm{~nm}$ low-temperature grown buffer layer on sapphire. Prior to the growth of the magnetic materials, the GaN surface was exposed to a $1: 1 \mathrm{HCl}: \mathrm{H}_{2} \mathrm{O}$ solution, followed by a $1: 99$ $\mathrm{HF}: \mathrm{H}_{2} \mathrm{O}$ solution for $2 \mathrm{~min}$ each with de-ionized water rinse between and after etching. ${ }^{20}$ Once loaded into the chamber, the substrates were annealed at $600{ }^{\circ} \mathrm{C}$ for $1 \mathrm{~h}$. Figures 2 (a) and 2(b) shows the RHEED patterns of a GaN(0001) surface just after annealing followed by cooling to RT for subsequent Fe growth. The streaky $(1 \times 1)$ patterns with clear Kikuchi lines suggest a smooth GaN surface. Fe films of a thickness $t_{\mathrm{Fe}}$ ranging from 3.0 to $6.0 \mathrm{~nm}$ were deposited by $e$-beam evaporation at RT at a rate of $2 \AA / \mathrm{min}$, and then oxidized to $\mathrm{Fe}_{3} \mathrm{O}_{4}$ by exposure to high-purity oxygen gas at an $\mathrm{O}_{2}$ partial pressure of $8 \times 10^{-4} \mathrm{mbar}$ at $230{ }^{\circ} \mathrm{C}$ for $10 \mathrm{~min}{ }^{10}$ After growth, the samples were retrieved from the growth chamber without a capping layer for subsequent ex situ characterizations. Thereafter, the nominal thicknesses of the predeposited Fe layers were quoted for the samples after the oxidation.

\section{RESULTS AND DISCUSSION}

The RHEED patterns along different azimuths for a 5.0 $\mathrm{nm} \mathrm{Fe}$ layer are shown in Figs. 2(c) and 2(d) and for the

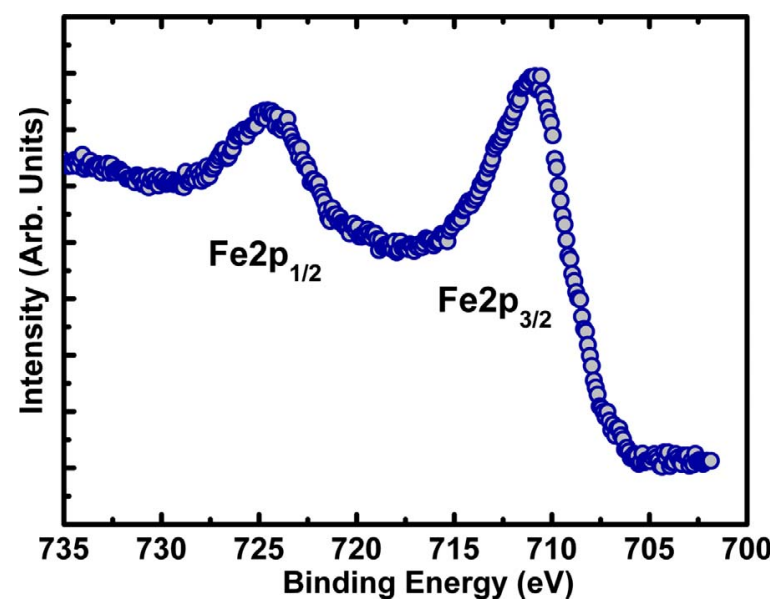

FIG. 3. (Color online) Fe $2 p$ core-level XPS spectrum obtained with normal incidence with respect to the surface of a $5.0 \mathrm{~nm}$ oxidized film using $\operatorname{Mg} K \alpha$ radiation.

oxidized layer in Figs. 2(e) and 2(f). The RHEED patterns of the Fe layer consist of a set of less intense reflections besides the main streaks of Fe. We attribute these features in the patterns to the formation of epitaxial domains of bcc Fe(110) with three different orientations on the hexagonal GaN substrates. This is similar to that reported for $\mathrm{Fe} / \mathrm{Al}_{2} \mathrm{O}_{3}(0001)$ by Shiratsuchi et al. ${ }^{21}$ The epitaxial relationship of the $\mathrm{Fe}$ film and $\mathrm{GaN}$ substrate as shown by the angular variation in the RHEED patterns in Figs. 2(c) and 2(d) is $(110)_{\mathrm{Fe}} \|(0001)_{\mathrm{GaN}}$ and $\langle 001\rangle_{\mathrm{Fe}} \|\langle 11 \overline{2} 0\rangle_{\mathrm{GaN}}$ with the Fe in bcc structure. Upon oxidation of the Fe films, both half- and first-order type streaks are present in Figs. 2(e) and 2(f), which are the characteristic patterns for the inverse spinel structure of $\mathrm{Fe}_{3} \mathrm{O}_{4}$. Comparing Figs. 2(e) and 2(f) with Figs. 1(a) and 1(b), the in-plane symmetries of the $\mathrm{Fe}_{3} \mathrm{O}_{4}$ layer are clearly the same as those of the $\mathrm{GaN}(0001)$ substrate and no rotation of the $\mathrm{Fe}_{3} \mathrm{O}_{4}$ lattice cell with respect to that of the $\mathrm{GaN}$ is observed. The intensity profile of the RHEED patterns gives an in-plane lattice-parameter $a_{\mathrm{Fe}_{3} \mathrm{O}_{4}}$ of $6.10 \AA$ for the $\mathrm{Fe}_{3} \mathrm{O}_{4}$ layer. This value matches well with the bulk $\mathrm{Fe}_{3} \mathrm{O}_{4}(111)$ value $a_{\text {bulk }}=5.96 \AA .^{22}$ We thus have an epitaxial $\mathrm{Fe}_{3} \mathrm{O}_{4}$ film with [111] axis perpendicular to the $\mathrm{GaN}(0001)$ surface and with epitaxial relationships $\quad \mathrm{Fe}_{3} \mathrm{O}_{4}(111)\langle 1 \overline{1} 0\rangle \| \mathrm{GaN}(0001)\langle 11 \overline{2} 0\rangle \quad$ and $\mathrm{Fe}_{3} \mathrm{O}_{4}(111)\langle 11 \overline{2}\rangle \| \mathrm{GaN}(0001)\langle 1 \overline{1} 00\rangle$, supporting our inferred atomic configuration (1) of Fig. 1(b).

One also needs to consider the possible existence of a metastable iron oxide phase $\gamma-\mathrm{Fe}_{2} \mathrm{O}_{3}$, which has almost the same inverse spinel structure as that of $\mathrm{Fe}_{3} \mathrm{O}_{4}$. It is, in practice, difficult to distinguish the two phases by RHEED patterns, which prompted us to characterize the films using XPS, probing the Fe valence state rather than its crystal structure. Figure 3 shows the Fe $2 p$ core-level spectrum of a $5.0 \mathrm{~nm}$ sample obtained at normal incidence with respect to the film surface using $\mathrm{Mg} K \alpha$ radiation. It is well established that peak positions and satellite structures in the XPS spectra are reliable means for identifying the state of Fe oxidation. From the figure, it is evidenced that the Fe $2 p_{3 / 2}$ and $\mathrm{Fe} 2 p_{1 / 2}$ peaks situated at around 711 and $724 \mathrm{eV}$ between which a 


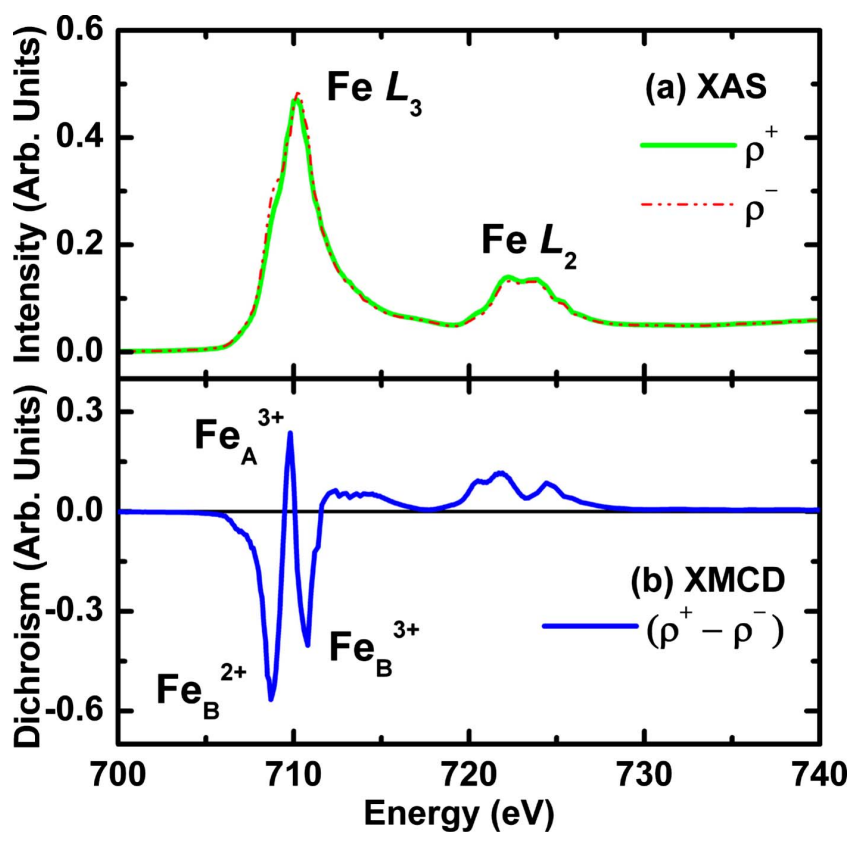

FIG. 4. (Color online) (a) Fe $L_{2,3}$ edge XAS and (b) XMCD spectra of a $5.0 \mathrm{~nm} \mathrm{Fe}_{3} \mathrm{O}_{4} / \mathrm{GaN}(0001)$ ultrathin film measured at RT. The labels A and B denote tetrahedral and octahedral sites, respectively, of the $\mathrm{Fe}$ ions.

board and structureless region can be identified, therefore suggesting the coexistence of the $\mathrm{Fe}^{3+}$ and $\mathrm{Fe}^{2+}$ cations. ${ }^{23,24}$ More importantly, no satellite can be identified at a binding energy of $\sim 719 \mathrm{eV}$, further eliminating the possible formation of $\gamma-\mathrm{Fe}_{2} \mathrm{O}_{3} .25$

We performed x-ray absorption spectroscopy (XAS) and XMCD measurements to further characterize the magnetite films. Figure 4 shows the Fe $L_{2,3}$ XAS and XMCD spectra of $\mathrm{Fe}_{3} \mathrm{O}_{4} / \mathrm{GaN}(0001)$ with $t_{\mathrm{Fe}}=5.0 \mathrm{~nm}$ taken at RT. The measurements, in total electron yield mode using circularly polarized X-rays, were performed in a portable octupole magnetic system at station 5U.1 of the Synchrotron Radiation Source at Daresbury Laboratory, U.K. The XMCD is the difference between the XAS spectra measured with a $0.6 \mathrm{~T}$ magnetic field applied parallel and antiparallel to the incident $\mathrm{X}$-ray beam. The characteristic ferrimagnetic multiplet structure of the Fe $L_{2,3}$ XAS and XMCD spectra, which features contributions from both $\mathrm{Fe}^{3+}$ cations at tetrahedral sites and $\mathrm{Fe}^{2+}$ and $\mathrm{Fe}^{3+}$ cations at octahedral sites, shows a good agreement with stoichiometric $\mathrm{Fe}_{3} \mathrm{O}_{4}{ }^{26,27}$ and, accordingly, provides direct evidence for the formation of $\mathrm{Fe}_{3} \mathrm{O}_{4}$ with a quality close to that of the bulk. It should be noted that, in the case of $\gamma-\mathrm{Fe}_{2} \mathrm{O}_{3}$, the peak corresponding to octahedral $\mathrm{Fe}^{2+}$ would be strongly suppressed. ${ }^{28,29}$

Magnetic hysteresis loops of the magnetite films measured by SQUID in the in-plane and out-of-plane geometry are depicted in Fig. 5. The external applied field was applied along the [1 $1 \overline{100}]$ axis in-plane and along the [0001] axis out-of-plane of the GaN film. All the films exhibit an easy in-plane magnetization due to the presence of shape anisotropy of the thin films. Also notable in the figure is the saturation of magnetization $M_{s}$ of the $5.0 \mathrm{~nm}$ sample at a field strength of $\sim 470 \mathrm{Oe}$. The coercivity $H_{c}$ of the $\mathrm{Fe}_{3} \mathrm{O}_{4}$ film

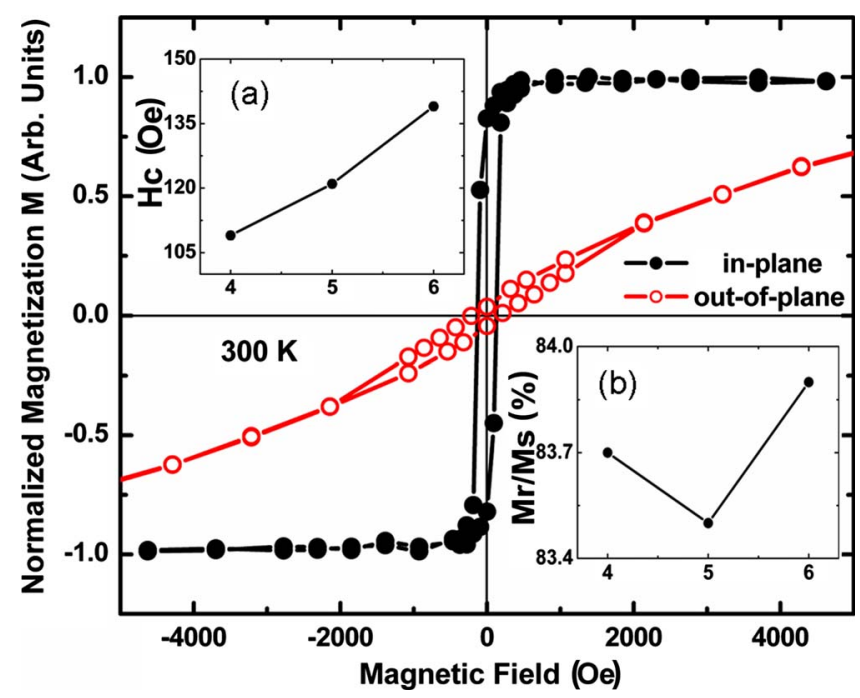

FIG. 5. (Color online) SQUID measurements of $5.0 \mathrm{~nm}$ $\mathrm{Fe}_{3} \mathrm{O}_{4} / \mathrm{GaN}(0001)$ at $\mathrm{RT}$. The magnetic field was applied along the [1] 100$]$ direction of the GaN film for the in-plane measurements. Inset (a) and (b) show, respectively, the thickness dependent coercivity and squareness of the hysteresis loop of the magnetite films. The $x$ axis of both insets represents the Fe thickness in $\mathrm{nm}$. The predetermined diamagnetic signals of the sapphire substrates have been subtracted from the data.

increases with increasing $t_{\mathrm{Fe}}$ as shown in inset (a) of Fig. 5; while in inset (b), the squareness, which is defined as $M_{r} / M_{s}$ remains fairly high and is largely constant within the range of $t_{\mathrm{Fe}}$ examined, indicating a good degree of magnetic ordering inside the films. The fast saturation behavior in our films is in contrast to the high-field magnetization slope observed in $\mathrm{Fe}_{3} \mathrm{O}_{4}$ films grown on $\mathrm{MgO}$, in which the epitaxial films are known to possess a high density of antiphase boundaries (APBs), thereby leading to an unsaturated magnetization at magnetic fields as high as $\sim 70 \mathrm{kOe} .{ }^{30}$ This suggests that the $\mathrm{Fe}_{3} \mathrm{O}_{4}$ films in the present study might be free from APBs or have a low density of such defects as we found previously in $\mathrm{Fe}_{3} \mathrm{O}_{4} / \mathrm{GaAs}(100)$ system. ${ }^{10}$

In order to gain insight into this issue, in-plane MR measurements of the magnetite films were performed in a standard four-probe configuration at RT. Electrical Ohmic contacts to the $\mathrm{Fe}_{3} \mathrm{O}_{4}$ films were made of thermally evaporated $50 \mathrm{~nm} \mathrm{Au}$ buffered with $10 \mathrm{~nm} \mathrm{Cr}$ into which the current that is along the [1 100$]$ direction of the $\mathrm{GaN}(0001)$ was applied. Such characterizations are reminiscences of the work delivered by Ziese and Blythe, in which the authors compared bulk and thin-film MR of $\mathrm{Fe}_{3} \mathrm{O}_{4} \cdot{ }^{31}$ Strikingly, they found abnormal electrical properties manifested as the high-field $\mathrm{MR}$ in the epitaxial films on $\mathrm{MgO}$, in contrast with the negligible MR in the bulk. This remarkable discrepancy has been attributed to the drastic alterations in electron transport in the $\mathrm{Fe}_{3} \mathrm{O}_{4}$ films with the APBs. ${ }^{31}$ Having recalled the above relevant report, we show in Fig. 6 the MR of a $4.0 \mathrm{~nm}$ oxidized film, from which a clear anisotropic MR with the film resistance in the longitudinal direction increases and saturates to a constant value above the saturation field when the magnetization is aligned with the external field is re- 


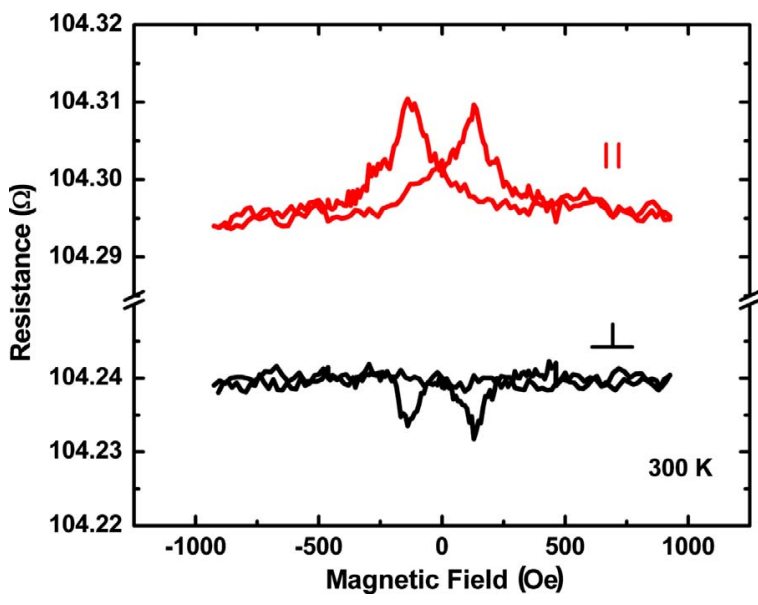

FIG. 6. (Color online) Longitudinal (upper curve) and transverse (lower curve) MR measurements of a $4.0 \mathrm{~nm}$ oxidized film acquired at RT. The applied current was along [1 $\overline{1} 00]$ crystallographic direction of $\mathrm{GaN}(0001)$.

vealed; The opposite is observed in the transverse geometry. Here, the MR is defined as $\left(R_{H c}-R_{H}\right) / R_{H}$ where $R_{H}$ and $R_{H c}$ denote, respectively, the resistance at $900 \mathrm{Oe}$ and at the $H_{c}$ of the film. The MR changes in the two geometries are, respectively, $0.0137 \%$ and $-0.0069 \%$. Notice that other film thicknesses also exhibit comparably small MR values, consistent with the very small MR effect of the $\mathrm{Fe}_{3} \mathrm{O}_{4}$ single crystal as mentioned. At this point, both SQUID and MR data support the hypothesis of a limited density of APBs in our oxide films. In particular, the latter results we have here are not in line with a belief of several former publications on reduced APB density in thicker magnetite films due to the larger $\mathrm{Fe}_{3} \mathrm{O}_{4}$ grain sizes that formed. ${ }^{32,33}$

The values of $M_{s}$ of the magnetite films with $t_{\mathrm{Fe}}$ spanning from 3.0 to $6.0 \mathrm{~nm}$ have also been extracted from the SQUID data and are shown in Fig. 7. The $M_{s}$ approaches that of the single-crystal value of $477 \mathrm{emu} \mathrm{cm}^{-3}$ with the thickness above $5.0 \mathrm{~nm} .{ }^{34}$ Considering the evolution of the $M_{s}$ with $t_{\mathrm{Fe}}$, we suggest that residual strain in the oxidized films may play a role in the $M_{s}$ reduction in the thinner films. It is arguable that the lowered values might be associated with parasitic phases like $\alpha-\mathrm{Fe}_{2} \mathrm{O}_{3}$ and $\mathrm{FeO}$, since the information depth by the $\mathrm{x}$-ray techniques used in this study did not probe the entire films. However, this appears unlikely because the presence of these phases should have altered the XMCD spectrum, as reported by $\mathrm{Lu}$ et $\mathrm{al} .{ }^{35}$ The present discussion can be, therefore, narrowed to either a $\mathrm{Fe}_{3} \mathrm{O}_{4}$ - $\mathrm{GaN}$ interface- or a strain-driven or both $M_{s}$ reduction mechanisms in the magnetic films with $t_{\mathrm{Fe}}$ less than $5.0 \mathrm{~nm}$, which is interesting for further investigations in the future.

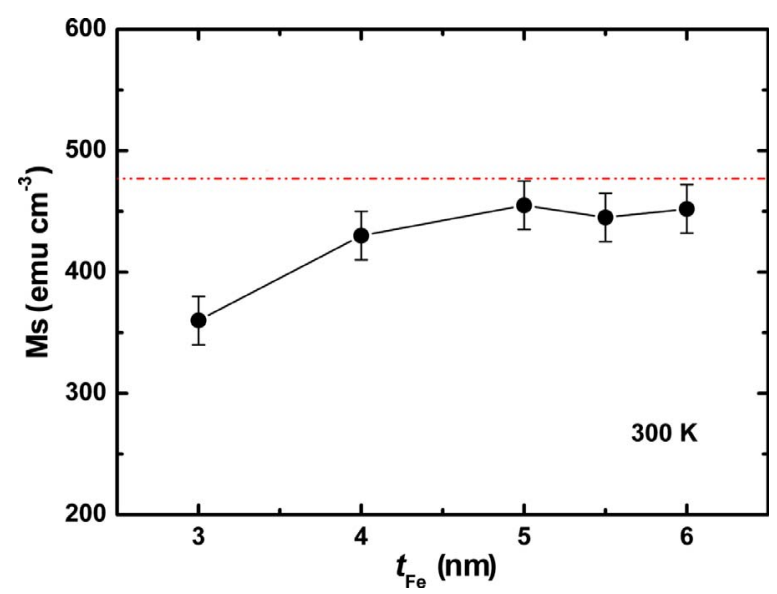

FIG. 7. (Color online) Thickness dependence of saturation magnetization $M_{s}$ of $\mathrm{Fe}_{3} \mathrm{O}_{4} / \mathrm{GaN}(0001)$ determined by SQUID at RT. The dotted line indicates the single-crystal value of $477 \mathrm{emu} \mathrm{cm}^{-3}$ (Ref. 34).

\section{CONCLUSIONS}

In summary, we have demonstrated the epitaxial growth of single-crystal ultrathin $\mathrm{Fe}_{3} \mathrm{O}_{4}(111)$ films on wide bandgap semiconductor $\mathrm{GaN}(0001)$ by postgrowth annealing of epitaxial ultrathin $\mathrm{Fe}$ films in an oxygen environment. The $\mathrm{Fe}_{3} \mathrm{O}_{4}$ unit cell shows no rotation with respect to that of the GaN substrate despite the $6.5 \%$ lattice mismatch. XAS and $\mathrm{XMCD}$ results reveal the characteristic ferrimagnetic coupling in $\mathrm{Fe}_{3} \mathrm{O}_{4}$. XPS and XMCD results eliminate the possible formation of metastable phase $\gamma-\mathrm{Fe}_{2} \mathrm{O}_{3}$. The fast saturation of the films at a field as low as 470 Oe shows that there is no evidence of APBs in our $\mathrm{Fe}_{3} \mathrm{O}_{4}$ thin films on $\mathrm{GaN}$. This is further supported by the negligible MR values found in the magnetotransport measurements. The slight deviation of the saturation magnetization from that of the single-crystal counterpart has been ascribed to an interface-, or a strain-driven or both mechanisms. These results might represent a significant step toward the development of functional magnetic oxide spintronic materials operational at room temperature that integrate with the intriguing properties of $\mathrm{GaN}$.

\section{ACKNOWLEDGMENTS}

The authors Y. B. X., W. Z., and G. L. thank the financial support from the STFC, UK. The experimental assistance from Sarah Thompson is also appreciated.

\footnotetext{
*Present address: MESA + Institute for Nanotechnology, University of Twente, P.O. Box 217, 7500 AE Enschede, The Netherlands. †yx2@ohm.york.ac.uk

${ }^{1}$ S. A. Wolf, D. D. Awschalom, R. A. Burham, J. M. Daughton, S. von Molnár, M. L. Roukes, A. Y. Chtchelkanova, and D. M.
}

Treger, Science 294, 1488 (2001).

${ }^{2}$ Spintronic Materials and Technology, edited by Y. B. Xu and S. M. Thompson (Taylor \& Francis, London, 2006).

${ }^{3}$ T. Dietl, H. Ohno, F. Matsukura, J. Cibert, and D. Ferrand, Science 287, 1019 (2000). 
${ }^{4}$ S. A. Chambers and R. F. C. Farrow, MRS Bull. 28, 729 (2003).

${ }^{5}$ R. A. de Groot, F. M. Mueller, P. G. van Engen, and K. H. J. Buschow, Phys. Rev. Lett. 50, 2024 (1983).

${ }^{6}$ C. Palmstrøm, MRS Bull. 28, 725 (2003).

${ }^{7}$ J. M. D. Coey and C. L. Chien, MRS Bull. 28, 720 (2003).

${ }^{8}$ Z. Zhang and S. Satpathy, Phys. Rev. B 44, 13319 (1991).

${ }^{9}$ Yu. S. Dedkov, U. Rüdiger, and G. Güntherodt, Phys. Rev. B 65, 064417 (2002).

${ }^{10}$ Y. X. Lu, J. S. Claydon, Y. B. Xu, S. M. Thompson, K. Wilson, and G. van der Laan, Phys. Rev. B 70, 233304 (2004).

${ }^{11}$ Y. B. Xu, E. Ahmad, J. S. Claydon, Y. X. Lu, S. S. A. Hassan, I. G. Will, and B. Cantor, J. Magn. Magn. Mater. 304, 69 (2006).

${ }^{12}$ M. Ferhat and K. Yoh, Appl. Phys. Lett. 90, 112501 (2007).

${ }^{13}$ R. J. Choudhary, S. Tiwari, D. M. Phase, R. Kumar, P. Thakur, K. H. Chae, and W. K. Choi, Appl. Phys. Lett. 92, 072102 (2008).

${ }^{14}$ S. Nakamura, T. Mukai, and M. Senoh, Appl. Phys. Lett. 64, 1687 (1994).

${ }^{15}$ S. Nakamura, M. Senoh, S. Nagahama, N. Iwasa, T. Yamada, T. Matsushita, H. Kiyoku, and Y. Sugimoto, Jpn. J. Appl. Phys. 35, L74 (1996).

${ }^{16}$ U. K. Mishra, Y.-F. Wu, B. P. Keller, S. Keller, and S. P. DenBaars, in Physics of Semiconductor Devices, edited by V. Kumar and S. K. Agarwal (India Norosa, Delhi, 1998), Vol. II, p. 878.

${ }^{17}$ B. Beschoten, E. Johnston-Halperin, D. K. Young, M. Poggio, J. E. Grimaldi, S. Keller, S. P. DenBaars, U. K. Mishra, E. L. Hu, and D. D. Awschalom, Phys. Rev. B 63, 121202(R) (2001).

${ }^{18}$ S. Krishnamurthy, M. van Schilfgaarde, and N. Newman, Appl. Phys. Lett. 83, 1761 (2003).

${ }^{19}$ M. Leszczynski, H. Teisseyre, T. Suski, I. Grzegory, M. Bockowski, J. Jun, S. Porowski, K. Pakula, J. M. Baranowski, C. T. Foxon, and T. S. Cheng, Appl. Phys. Lett. 69, 73 (1996).

${ }^{20}$ L. L. Smith, S. W. King, R. J. Nemanich, and R. F. Davis, J. Electron. Mater. 25, 805 (1996).

${ }^{21}$ Y. Shiratsuchi, Y. Endo, M. Yamamoto, and S. D. Bader, J. Appl. Phys. 97, 10J106 (2005).
${ }^{22}$ C. N. Rao and B. Raveau, Transitional Metal Oxides (VCH, New York, 1995).

${ }^{23}$ C. Ruby, B. Humbert, and J. Fusy, Surf. Interface Anal. 29, 377 (2000).

${ }^{24}$ T. Fujii, F. M. F. de Groot, G. A. Sawatzky, F. C. Voogt, T. Hibma, and K. Okada, Phys. Rev. B 59, 3195 (1999).

${ }^{25}$ S. Gota, E. Guiot, M. Henriot, and M. Gautier-Soyer, Phys. Rev. B 60, 14387 (1999).

${ }^{26}$ P. Morrall, F. Schedin, G. S. Case, M. F. Thomas, E. Dudzik, G. van der Laan, and G. Thornton, Phys. Rev. B 67, 214408 (2003).

${ }^{27}$ D. J. Huang, C. F. Chang, H.-T. Jeng, G. Y. Guo, H.-J. Lin, W. B. $\mathrm{Wu}, \mathrm{H}$. C. Ku, A. Fujimori, Y. Takahashi, and C. T. Chen, Phys. Rev. Lett. 93, 077204 (2004).

${ }^{28}$ L. Signorini, L. Pasquini, F. Boscherini, E. Bonetti, I. Letard, S. Brice-Profeta, and Ph. Sanctavit, Phys. Rev. B 74, 014426 (2006).

${ }^{29}$ E. Pellegrain, M. Hagelstein, S. Doyle, H. O. Moser, J. Fuchs, D. Vollath, S. Schuppler, M. A. James, S. S. Saxena, L. Niesen, O. Rogojanu, G. A. Sawatzky, C. Ferrero, M. Borowski, O. Tjernberg, and N. B. Brookes, Phys. Status Solidi 215, 797 (1999) b.

${ }^{30}$ D. T. Margulies, F. T. Parker, F. E. Spada, R. S. Goldman, J. Li, R. Sinclair, and A. E. Berkowitz, Phys. Rev. B 53, 9175 (1996).

${ }^{31}$ M. Ziese and H. J. Blythe, J. Phys.: Condens. Matter 12, 13 (2000).

${ }^{32}$ W. Eerenstein, T. T. M. Palstra, T. Hibma, and S. Celotto, Phys. Rev. B 66, 201101 (2002).

${ }^{33}$ J.-B. Moussy, S. Gota, A. Bataille, M.-J. Guittet, M. GautierSoyer, F. Delille, B. Dieny, F. Ott, T. D. Doan, P. Warin, P. Bayle-Guillemaud, C. Gatel, and E. Snoeck, Phys. Rev. B 70, 174448 (2004)

${ }^{34}$ S. Okamoto and K. Kohn, Magnetic Ceramics (Gihodo, Japan, 1986), p. 84.

${ }^{35}$ Y. X. Lu, J. S. Claydon, E. Ahmad, Y. B. Xu, S. M. Thompson, K. Wilson, and G. van der Laan, IEEE Trans. Magn. 41, 2808 (2005). 